\title{
Reutter, Werner (2020): Die deutschen Länder. Eine Einführung
}

\author{
Wiesbaden: Springer VS. 166 Seiten. 27,99€
}

\author{
Henrik Scheller $\mathbb{D}$
}

Angenommen: 29. Juni 2021 / Online publiziert: 19. Juli 2021

(C) Der/die Autor(en) 2021

$\mathrm{Zu}$ den scharfsinnigen Beobachtern der deutschen Föderalismusforschung zählt seit Jahren Werner Reutter. Er fällt dabei immer wieder durch sein Gespür für Themen, Institutionen und Perspektiven auf, mit denen er wichtige Beiträge zur Funktionsweise aus dem Maschinenraum des deutschen Bundesstaates liefert, die sich meist jenseits der ausgetretenen Diskussionspfade bewegen. Mit diesem Gegen-den-StrichBürsten ringt Reutter dem ohnehin etwas stiefmütterlich gepflegten Untersuchungsgegenstand oft neue Erkenntnisse und Impulse ab. Auch seine kleine Einführung „Die deutschen Länder“, die - laut Klappentext - „nur“ als Lehrbuch für politisch Interessierte, Lehrende und Studierende der einschlägigen Fächer verkauft wird, basiert auf einem solchen Perspektivwechsel mit Ansage. Denn die Analyse der deutschen Bundesstaatskonstruktion mit ihren charakteristischen Formen der Politikgestaltung wird hier konsequent Bottum-up aus Sicht der Länder vorgenommen. Auf diese Weise will Reutter einen klaren Gegenakzent zu den einschlägigen Untersuchungen und der veröffentlichten Meinung setzen, die die kooperative Einheitsbildung bzw. deren Verhinderung problematisieren. Es geht mithin um eine Darstellung der Vielfalt, die zwar in der theoretisch oft bemühten Formel von der ,Vielfalt in der Einheit" Würdigung findet, durch eine starke Fokussierung der Effizienz-Dimension in den einschlägigen Politikverflechtungs(fallen)-Ansätzen allerdings nur allzu oft desavouiert wird. In den Mittelpunkt seiner Darstellungen rückt Reutter stattdessen die Frage, „ob und inwieweit der Bundesstaat in den Ländern die Voraussetzungen findet für ,politische Selbstbestimmung', d.h. für Demokratie“ (S. 6). Damit sollen zugleich die Möglichkeiten und Grenzen der Landespolitik aufgezeigt werden, die - gemäß einschlägiger Bevölkerungsbefragungen zum Thema - für viele Menschen eher unter der Wahrnehmungsschwelle firmiert.

Henrik Scheller $(\bowtie)$

Deutsches Institut für Urbanistik, Berlin, Deutschland

E-Mail: scheller@difu.de 
Nach seinem einführenden Problemaufriss wendet sich Reutter sogleich der historischen Genese der deutschen Länder zu. Dies ist insofern erhellend, da es ihm gelingt, nicht nur - wie in anderen Lehrbüchern dieser Art - die konträren Föderalismusvorstellungen der Alliierten und der Volksparteien nach $1945 \mathrm{zu}$ illustrieren. Vielmehr zeichnet er die unterschiedlichen Entwicklungen innerhalb der einzelnen Besatzungszonen einschließlich der SBZ kurz nach und verweist dann vor allem auf die prägende Mitwirkung der Länder in den verfassungsgebenden Debatten von Herrenchiemsee und im Parlamentarischen Rat. Mit Blick auf die Länder als „Kunstgebilde“ (S. 21) und die Frage einer möglichen Neugliederung nach Art. 29 GG scheut Reutter - wie punktuell auch im gesamten Bändchen - keine klare Wertung. Die demografischen, ökonomischen und kulturellen Differenzen zwischen den Ländern werden bereits in diesem Abschnitt durch tabellarische Synopsen veranschaulicht.

Eine theoretische Kontextualisierung seines Untersuchungsgegenstandes liefert Reutter erst im dritten Abschnitt, in dem er die verfassungsrechtlichen Grundlagen und politikwissenschaftliche Erklärungsansätze gemeinsam problematisiert. Diese Kapitelanordnung verdeutlicht den Lehrbuchcharakter. Gleichwohl wäre hier zu überlegen gewesen, ob nicht gerade mit Blick auf Studierende ein eigener, abgeschlossener Abschnitt zu den einschlägigen Theorien für mehr strukturelle Klarheit gesorgt hätte. Dann hätte man womöglich auch noch die ökonomisch inspirierten Föderalismustheorien würdigen können, die den deutschen Diskurs zum Teil dominieren. Die Sorge bestand hier aber wohl darin, einen zu großen Perspektivwechsel - von den Ländern weg - vornehmen zu müssen, da die einschlägigen Theorien immer das Verhältnis zwischen Bund und Ländern problematisieren. Nichtsdestotrotz enthält die gewählte Argumentationsführung einen wichtigen impliziten Hinweis auf die notwendigen analytischen Dimensionen der Föderalismusproblematik: Ohne eine Würdigung der verfassungsrechtlich normierten und durch die Ewigkeitsklausel geschützten Grundentscheidungen für die bundesstaatliche Ordnung muss auch eine politikwissenschaftliche Theoriebildung zum Mehrebenensystem rudimentär bleiben.

Eigene Kapitel widmet Reutter - den in der Einleitung formulierten Vermittlungsanspruch einlösend - folgerichtiger Weise der repräsentativen und direkten Demokratie sowie den Parteiensystemen in den Ländern. Nicht nur mithilfe verschiedener tabellarischer Gegenüberstellungen gelingt es Reutter auf diese Weise die Variationsbreite an institutionellen Ausprägungen, Regelungen und Entwicklungstendenzen in den Ländern knapp und konzise aufzuzeigen. Dabei kommt er zu dem Schluss, dass von einer „Entkopplung“ (S. 91) der Parteiensysteme von Bund und Ländern nicht gesprochen werden könne, dass sich gleichwohl eine Regionalisierung beobachten lasse. Mit einem separaten Abschnitt zu den Landesparlamenten widmet sich Reutter einem Themenfeld, das er bereits seit Jahren intensiv beforscht. Kaum verwunderlich ist es deshalb, dass er sich hier sehr klar und pointiert gegen die ewigen Parlamentarismus-Polemiker vom Schlage eines Hans-Herbert von Arnims stellt. So seien die Landesparlamente eben nicht nur Anstalten zur Sicherung von Pfründen einer politischen Klasse, sondern - mit Verweis auf Werner Patzelt vielmehr eine ,zusätzliche Ebene politischer Responsivitätsentfaltung, Mitsteuerung und politischer Führung“" (S. 95). Um diese Perspektive zu unterstreichen, kann sich Reutter die Feststellung nicht verkneifen, dass sich ,pauschale Urteile“ (S. 95) hier 
verböten. Angesichts so mancher unreflektierten, weil vielleicht auch unerfahrenen und nicht hinreichend im Seminarraum geübten Debattenbeiträge von Studierenden scheint ein solcher Hinweis - gerade in einem Lehrbuch - durchaus angemessen und hilfreich. Dies gilt umso mehr, da Reutter im gesamten Band die Breite der theoretischen Positionen - wenn auch skizzenhaft - abbildet und damit in bester Tradition dem Kontroversitätsgebot des Beutelsbacher Konsenses folgt.

Mit seiner Einführung „Die deutschen Länder“ ist Reutter ein kurzweiliges Bändchen gelungen, das den Facettenreichtum des in den Mittelpunkt gestellten Untersuchungsgegenstands kenntnisreich und pointiert beleuchtet. Dabei hält die Darstellung durchaus auch das eine oder andere Detail für diejenigen bereit, die sich schon seit Jahren mit der Materie befassen - seien es beispielsweise die Problematik der Einspruchsgesetze, die stets im Schatten der vermeintlich blockadeförderlichen Zustimmungsgesetze stehen, die Landesverfassungsgerichte als kaum wahrgenommene Institutionen oder die ewige Spannungslage zwischen Föderalismus- und Demokratieprinzip. Reutter führt mit seinen anschaulichen und konkreten Darstellungen den Nachweis, dass dieser Föderalismus lebt und - manchmal vielleicht etwas zu geräuschlos - wie ein Uhrwerk arbeitet.

Funding Open Access funding enabled and organized by Projekt DEAL.

Open Access Dieser Artikel wird unter der Creative Commons Namensnennung 4.0 International Lizenz veröffentlicht, welche die Nutzung, Vervielfältigung, Bearbeitung, Verbreitung und Wiedergabe in jeglichem Medium und Format erlaubt, sofern Sie den/die ursprünglichen Autor(en) und die Quelle ordnungsgemäß nennen, einen Link zur Creative Commons Lizenz beifügen und angeben, ob Änderungen vorgenommen wurden.

Die in diesem Artikel enthaltenen Bilder und sonstiges Drittmaterial unterliegen ebenfalls der genannten Creative Commons Lizenz, sofern sich aus der Abbildungslegende nichts anderes ergibt. Sofern das betreffende Material nicht unter der genannten Creative Commons Lizenz steht und die betreffende Handlung nicht nach gesetzlichen Vorschriften erlaubt ist, ist für die oben aufgeführten Weiterverwendungen des Materials die Einwilligung des jeweiligen Rechteinhabers einzuholen.

Weitere Details zur Lizenz entnehmen Sie bitte der Lizenzinformation auf http://creativecommons.org/ licenses/by/4.0/deed.de. 